\title{
Short-term outcomes of single event multilevel surgery for children with diplegia in a South African setting
}

\author{
Wood L1, Firth GB², Potterton $\mathrm{J}^{3}$ \\ ${ }^{1}$ BSc Physio (Wits), MSc Physio (Wits); Department of Physiotherapy, University of the Witwatersrand \\ ${ }_{2}$ MBBCh, FCS(Orth)SA, MMed(Orth); Orthopaedic Surgeon, Chris Hani Baragwanath Academic Hospital, Department of Orthopaedic Surgery, University of \\ the Witwatersrand \\ ${ }^{3} \mathrm{PhD}$; Associate Professor, Department of Physiotherapy, University of the Witwatersrand
}

Corresponding author: Dr GB Firth, University of the Witwatersrand, 7 York Road, Johannesburg, 2193, Gauteng, South Africa; email: greg.firth@gmail.com; tel: +27826633299

\begin{abstract}
Background: Although single event multilevel surgery (SEMLS) is well supported in the literature for walking children with cerebral palsy (CP) there is little evidence to show the outcomes in developing countries with limited resources. Further there is no literature reporting on the use of SEMLS in walking children with HIV encephalopathy (HIVE). The primary aim of this study was to investigate whether SEMLS can have good short-term outcomes in a South African setting, and the secondary aim was to compare the outcomes of SEMLS in children with diplegia secondary to CP and HIVE.

Methods: A prospective cohort study of ten children with spastic diplegia was enrolled (six with CP, four with HIVE) and followed up for 12 months. All children underwent SEMLS and received peri-operative therapy at a local clinic, hospital or a special-needs school. The primary outcome measures were the Edinburgh Visual Gait Score (EVGS), Gross Motor Function Measure-66 (GMFM-66) and the Functional Mobility Scale (FMS) measured pre-operatively (T1), at six months (T2) and at one year (T3).

Results: There was an overall mean improvement of 6.4 in the EVGS and 3.2\% in the GMFM-66 at the one-year follow-up assessment. The FMS revealed an initial deterioration in function at six months, with return to pre-operative function at the one-year assessment. Improvements in the GMFM-66 were found to be clinically significant. When comparing children with CP to those with HIVE the improvements were similar.

Conclusion: The results of this study indicate that the early outcomes of SEMLS in a South African setting, with scarce resources, are similar to those seen in developed countries. It may also be possible to use the same SEMLS treatment principles seen in the management of children with CP for children with static HIVE. Further follow-up is however needed in both of these areas
\end{abstract}

Level of evidence: Level 4

Key words: short-term outcomes, single event multilevel surgery, children, diplegia

Citation: Wood L, Firth GB, Potterton J. Short-term outcomes of single event multilevel surgery for children with diplegia in a South African setting. SA Orthop J 2018;17(2):44-48. http://dx.doi.org/10.17159/2309-8309/2018/v17n2a8

Editor: Dr LC Marais, University of KwaZulu-Natal

Received: May 2017

Accepted: October 2017

Published: May 2018

Copyright: () 2018 Wood L. This is an open-access article distributed under the terms of the Creative Commons Attribution Licence, which permits unrestricted use, distribution and reproduction in any medium, provided the original author and source are credited.

Funding: This study was partially funded by a grant awarded to Joanne Potterton by the National Research Foundation of South Africa (NRF).

Conflict of interest: All authors declare that they have no conflict of interest with respect to the writing of this article. 


\section{Introduction}

Single event multilevel surgery (SEMLS) is the current preferred orthopaedic management of ambulant children with cerebral palsy $(\mathrm{CP})$ in developed countries. ${ }^{1-4}$ SEMLS by definition is when two or more orthopaedic procedures are being performed on the musculoskeletal system, at more than one level, at the same time. ${ }^{2,5,6}$ The benefits of SEMLS include one hospital admission, one rehabilitation period and the prevention of further secondary deformities. ${ }^{6}$

Bearing in mind that the natural progression for the walking child with $\mathrm{CP}$ is a regression in gross motor function, ${ }^{7,8}$ the aim of SEMLS is to improve gait efficiency and appearance, gross motor function, independence and quality of life ${ }^{9}$ and to maintain the child's ability to walk into adulthood. SEMLS does not improve the gross motor function classification system (GMFCS) of the child but attempts to maintain it into adulthood. ${ }^{1,2}$ Current literature reveals that the gait, functional ability and quality of life does improve at a one- and fiveyear follow-up after SEMLS 9,10 despite an initial regression in GMFCS level at three months post-surgery. ${ }^{11}$

With the majority of the research being conducted in developed countries there is little evidence to support the value of SEMLS in children with CP in developing countries. The discrepancy in resources, namely financial, skills and therapy, available in developing countries in comparison to developed countries leads to the need to investigate the effect that this paucity of resources has on the outcomes of the surgery. The lack of resources can lead to the late diagnosis and treatment of children with $\mathrm{CP}$ resulting in an altered clinical picture with increased contractures and secondary complications. ${ }^{12}$ The evidence for successful outcomes of SEMLS in developing countries is limited. ${ }^{12}$

The high prevalence of human immunodeficiency virus (HIV) in sub-Saharan Africa ${ }^{13-15}$ and the subsequent development and use of antiretroviral therapy (ART) has resulted in a population of children with associated sequelae who have previously not been treated and managed. HIV encephalopathy (HIVE) is the most commonly seen neurological complication in HIV. ${ }^{16}$ HIV is neurotrophic, and infected monocytes are able to cross the immature blood brain barrier. The central nervous system (CNS) may be infected in utero, perinatally or postnatally. Unlike CP, damage to the CNS is then progressive as the virus replicates. The most clinically useful definition of HIVE is the failure to gain milestones, or the loss of previously acquired milestones or cognitive abilities verified by standardised developmental and neuropsychological scales. ${ }^{17}$ Children with HIVE frequently present with bilateral hyperreflexia. ${ }^{17}$ Many children with HIVE on ART present with a static encephalopathy, and clinically present very similarly to those with spastic diplegic CP. ${ }^{13}$ These patients with HIVE are often weaker and present with a jump gait pattern in our experience. Despite the similar clinical presentation between the two groups there is no evidence showing the appropriate surgical management of children with HIVE using SEMLS techniques.

The primary aim of this study was to investigate whether SEMLS could have good short-term outcomes in a South African setting, and the secondary aim was to compare the outcomes of SEMLS in children with diplegia secondary to CP and HIVE. Taking into account the high prevalence of HIVE in the population of children presenting with spastic diplegia at large academic hospitals in South Africa, we determined that this population needed to be included in the study in order to get a true reflection of the population served by the hospital and to see what the outcomes of SEMLS were for this population of children presenting with similar clinical features to the children with CP diplegia.

\section{Materials and methods}

The parents of all the children gave their consent for them to participate in the study. Ethics approval was granted by the Human Research and Ethics Committee and the local Hospital Board
(Clearance number M120907). All children and their parents were given information sheets with an attached consent and assent form. A cohort of the first ten patients was included in the study.

\section{Participants}

All children referred by the surrounding clinics, special needs schools and primary health care facilities in different districts presenting with spastic diplegic CP or spastic diplegia as a result of HIVE with GMFCS Level II or III, were assessed for inclusion in the study. Children were included if they were between the ages of 6 and 18 years, were able to follow two-part instructions and could walk at least $10 \mathrm{~m}$ with or without an assistive device. Furthermore, they were required to be able to attend weekly follow-ups at their regular place of therapy post surgery. Children were excluded if they presented with hemiplegic or quadriplegic CP, if they had an unconfirmed diagnosis or if they had received orthopaedic surgery or botulinum toxin within the previous six months. Children were also excluded if they were HIV positive, and had not been receiving ART for at least one year, or if they their CD 4 count was less than 300 , or if they had high viral loads, above 1000 copies $/ \mathrm{ml}$.

Pre-operatively six children were GMFCS level II (one HIV positive) and four children were GMFCS level III (three HIV positive). The mean age at the time of surgery was nine years ten months $(S D=$ two years four months, range 6-14 years). There were 5.8 surgical procedures per patient on average. Fifty-eight procedures were performed, which included 14 proximal femur derotation osteotomies, two psoas lengthenings, eight adductor releases, 18 medial hamstring lengthenings, eight semi-tendinosis transfers, six Strayer procedures and two metatarsophalangeal joint fusions.

\section{Outcome measures}

Assessments on all children were done at three time intervals: preoperatively (T1), at six months (T2) and one year post-operatively (T3). The Edinburgh Visual Gait Score (EVGS), which is valid and reliable, ${ }^{18,19}$ was used to assess the gait. The tool is scored according to the deviation of the gait pattern from normal. The gait pattern is assessed on videos taken from both the coronal and sagittal views. A greater EVGS score indicates a larger deviation from normal gait. The videos were copied onto a disk and given to a second orthopaedic surgeon, with more than five years' experience, to score according to the EVGS guidelines. The date at which the video was taken was concealed and the videos were not in chronological order.

The Gross Motor Function Measure (GMFM-66) was selected as it is the gold standard for the assessment of gross motor function in children with $\mathrm{CP} .{ }^{20}$ The more functional the child is, the higher their GMFM score will be. It is valid, reliable, and able to detect change after an intervention. ${ }^{8,20,21}$ All assessments were done by the same physiotherapist with more than five years experience at T1, T2 and T3.

The Functional Mobility Scale (FMS) was used to assess the daily functional mobility of the children. As with the GMFM, a high score indicates greater function. It was scored according to parental report by the same physiotherapist at T1, T2 and T3. It has been proven to have good construct validity and is able to detect change after an intervention. ${ }^{11}$

\section{Statistical analysis}

Due to the small sample size the data was analysed descriptively, using the minimum clinically important difference (MCID) as per the literature to determine whether the results showed clinical significance for each of the outcome scoring tools. MCID is a threshold for determining when meaningful changes occur. ${ }^{22}$ Although the original article on the EVGS gave an improvement of 4 as significant, we used 
the MCID for the EVGS as described by Gupta et al. They showed that a figure of 11 at the six-month post-operative assessment and 15 at the one-year post-operative assessment (T3) was a more significant MCID. ${ }^{23,24}$ The MCID in the GMFM-66 after intervention is $1.5 .{ }^{21}$

\section{Results}

There were ten participants in this prospective cohort study (six females, four males). Six children had spastic diplegic CP and four children had HIVE. All children with HIVE were receiving ART, and were adherent with treatment. The mean baseline CD4 count was 1757.25 cells $/ \mathrm{mm}^{3}$ (range $795-3$ 024). Two of the baseline viral loads were not detectable, one specimen was rejected, and one was 540 copies.

Five children attended physiotherapy within the government health care setting, either at a clinic or at a hospital, four children attended local special needs schools where they received physiotherapy, and one child received therapy from a volunteer physiotherapist in the community. All children received the same additional homebased exercise programme. Children receiving physiotherapy at the schools had weekly physiotherapy, except during school holidays. Children receiving therapy in the government health care setting or the community received weekly physiotherapy initially for six weeks, then monthly therapy thereafter.

There was one adverse event noted from the surgery. The child's left femoral nerve was partially cut which resulted in a partial loss of sensation in the leg, generalised weakness, especially with regard to hip flexion and knee extension, and severe pain. A repair was done and she was given a TENS machine to manage her pain. At the oneyear follow-up period her pain was being fully managed with a TENS machine and some of her sensation had returned to her leg. She still had generalised weakness in her leg and was using a brace to assist with ambulation. Due to this she was unable to ambulate $10 \mathrm{~m}$ at

Table I: Summary of the changes in the EVGS

\begin{tabular}{|l|c|c|c|}
\hline & $\begin{array}{c}\text { Change from } \\
\text { T1 to T2 }\end{array}$ & $\begin{array}{c}\text { Change from } \\
\text { T2 to T3 }\end{array}$ & $\begin{array}{c}\text { Change from } \\
\text { T1 to T3 }\end{array}$ \\
\hline Total & $\mathbf{6 . 8}$ & $\mathbf{- 0 . 3}$ & $\mathbf{6 . 5}$ \\
\hline Foot & 1.2 & 0.9 & 2.2 \\
\hline Knee & 3.4 & -0.9 & 2.5 \\
\hline Hip & 0.9 & 0.3 & 1.2 \\
\hline Pelvis & 0.3 & -0.3 & 0.0 \\
\hline Trunk & 0.9 & -0.3 & 0.6 \\
\hline
\end{tabular}

Table II: GMFM-66 scores at each assessment

\begin{tabular}{|l|c|c|c|}
\hline \multirow{2}{*}{} & \multicolumn{3}{|c|}{ GMFM-66 } \\
\hline 1 & T1 & T2 & T3 \\
\hline 2 & 69.63 & 72.16 & 74.16 \\
\hline 3 & 80.93 & 79.11 & 92.05 \\
\hline 4 & 50.09 & 44.56 & 46.5 \\
\hline 5 & 82.99 & 89.7 & 92.05 \\
\hline 6 & 71.22 & 61.51 & 65.33 \\
\hline 7 & 76.75 & 69.63 & 74.16 \\
\hline 8 & 72.63 & 69.63 & 70.04 \\
\hline 9 & 52.09 & 53.38 & 55.15 \\
\hline 10 & 50.85 & 50.62 & 53.86 \\
\hline Mean & 69.63 & 67.75 & 75.34 \\
\hline SD & $\mathbf{6 7 . 6 8}$ & $\mathbf{6 5 . 8 1}$ & $\mathbf{6 9 . 8 6}$ \\
\hline
\end{tabular}

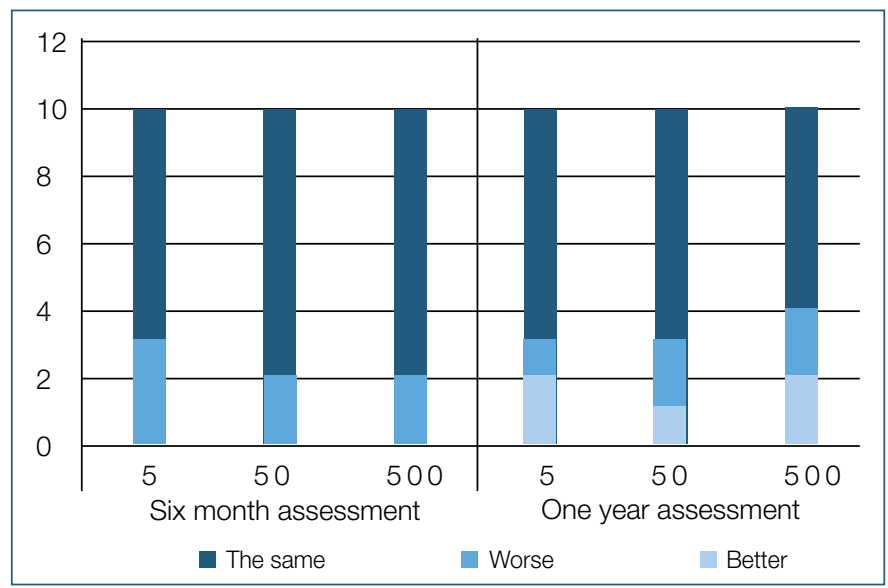

Figure 1. Summary of the change seen in the FMS

both the six-month and one-year post-operative assessments and therefore her EVG scores were not included in the data.

The EVGS showed a mean improvement from $\mathrm{T} 1$ to $\mathrm{T} 2$ of 6.8 , with a final improvement of 6.5 at T3. As illustrated in Table / the greatest improvements in the gait pattern were seen at the foot and knee, with smaller improvements occurring at the hip, pelvis and trunk. Deterioration from T2 to T3 occurred at the knee, pelvis and trunk with the improvement continuing at the foot and hip at T3. Although the original article on the EVGS gave an improvement of 4 as a significant MCID, the MCID for the EVGS as described by Gupta et al. is 11 at six months and 15 at one-year post-operative assessment (T3).23,24 The change in EVGS in the current study, although an improvement, was not clinically significant.

A summary of the GMFM-66 scores can be seen in Table II. The GMFM-66 scores ranged from 44.56 to 92.05 over the three assessments. There was a $1.87(2.77 \%)$ decrease in the mean GMFM-66 scores at T2. There was, however, an overall mean improvement of 2.18 (3.23\%) from T1 to T3. In six patients the GMFM-66 scores improved between $\mathrm{T} 1$ and $\mathrm{T} 3$, and four scores decreased (one of these was HIV positive). The changes seen in the GMFM-66 were clinically significant as the reported MCID for the GMFM-66 is $1.5 .^{21}$

The overall change in the FMS at T2 and T3 is illustrated in Figure 1. At T2, there was an overall decline in functional mobility, with two to three children needing more assistance during gait for each distance. At the one-year follow-up period, two children were mobilising with less assistance for each distance. The changes in functional gait using the FMS score corresponded with the changes seen in the GMFM-66. Regarding the FMS at $500 \mathrm{~m}$ at one-year follow-up, two children were worse (one with the femoral nerve injury), two were better and the remaining six were unchanged.

Table III summarises differences between the children with CP and those with HIVE at baseline. The children with $\mathrm{CP}$ were more functional than those with HIVE. The GMFCS levels correspond with the pre-operative EVGS and GMFM-66 scores. The children with CP had mean scores of 30.66 and 79.14 for the EVGS and GMFM-66 respectively, whereas the mean scores for the HIVE group were 41 and 69.3.

The overall changes post-operatively seen in children with CP and those with HIVE were very similar. The children with HIVE showed

Table III: Demographics of the children with CP and HIVE

\begin{tabular}{|l|c|c|c|} 
& $\begin{array}{c}\text { Age mean } \\
\text { (SD) }\end{array}$ & Sex & GMFCS level \\
\hline $\begin{array}{l}\text { CP } \\
(n=6)\end{array}$ & $\begin{array}{c}\text { 10 yrs 11 months } \\
(26.97)\end{array}$ & $F=3$ & $\|=5$ \\
\hline HIVE & 8yrs 4 months & $F=3$ & $\|=1$ \\
$(n=4)$ & $(32.71)$ & $M=1$ & $\|=1$ \\
\hline & & & $\|=3$ \\
\hline
\end{tabular}


Table IV: Summary of changes seen in each assessment tool for children with CP and HIVE

\begin{tabular}{l|c|c|c|c|} 
& & EVGS & GMFM-66 & $\begin{array}{c}\text { FMS } \\
(5 \mathrm{~m} / 50 \mathrm{~m} / 500 \mathrm{~m})\end{array}$ \\
\hline $\begin{array}{l}\text { Change from } \\
\text { T1 to T2 }\end{array}$ & CP & 7.40 & -2.49 & $-2 /-1 /-2$ \\
\hline Change from & CPVE & 6.00 & -0.95 & $-1 /-1 / 0$ \\
\hline T2 to T3 & HIVE & -1.00 & 4.60 & $1 / 0 / 0$ \\
\hline Change from & CP & 6.40 & 3.25 & $3 / 1 / 2$ \\
\hline T1 to T3 & HIVE & 6.50 & 2.11 & $-1 /-1 /-2$ \\
\hline & & & & $2 / 0 / 2$
\end{tabular}

less functional deterioration at T2, with respect to both the GMFM-66 and the FMS. The children with HIVE did have a greater improvement in the FMS at T3. With regard to gait as measured by the EVGS, the gait continued to improve in the children with HIVE from T2 to T3; however, the children with $\mathrm{CP}$ showed a small deterioration in gait leading up to T3. Table $\mathrm{IV}$ shows a summary of these results.

\section{Discussion}

SEMLS is widely used in the orthopaedic management of children with CP. There is substantial research showing positive outcomes in these children in developed countries. The aim of this study was to gain insight into the outcomes of children post SEMLS in a developing country where the resources and population differ immensely from the developed world.

Gait analysis to determine outcomes post SEMLS shows an improvement in gait up to the one-year follow-up period. ${ }^{22}$ These results have been found to be maintained up to the five-year followup period. ${ }^{1}$ This study yielded similar results with an improvement of the mean EVG scores at the one-year follow-up. In the current study, the mean change in EVGS was 6.5 from T1 to T3, with a deterioration of 0.3 from T2 to T3. However, results were not clinically significant as the recorded MCID for the EVGS for six-month and one-year followup are 11 and 15 respectively. ${ }^{25}$ This could be due to the limited supervised rehabilitation received post-surgery, with the majority of the children receiving weekly therapy for the first six weeks, then only monthly check-ups thereafter by different physiotherapists with varying experience.

An initial deterioration in function was seen at T2 with an overall improvement at T3 of 2.13. The MCID for the GMFM-66 of a large effect size $(0.8)$ is $1.5,{ }^{24}$ therefore it can be determined that the change seen was clinically significant. Greater improvement was seen in the GMFM-66 in the current study in contrast to the prospective study done by Thomason et al., ${ }^{1}$ where the mean change in GMFM-66 at one year was only 0.2 . This result could be due to the fact that the children in developing countries are often diagnosed late, receive limited physiotherapy and have more secondary complications. This may lead to them not reaching their full functional potential prior to surgery, which in turn may result in a greater potential to change post-operatively.

The results yielded by the FMS were very similar to those of the GMFM-66. There was an initial functional deterioration seen at T2 and there was minimal change from T1 to T3. Although this differs slightly from the GMFM-66 results, it coincides with the literature. It was found that the changes in the FMS could also have been attributed to incorrect prescription of walking aids prior to surgery. This is applicable in poor resource settings, where there are limited funds available for devices such as wheelchairs that will only be used occasionally.

The sample size was too small to directly compare the outcomes of the children with CP to those with HIVE. However, as the first study of its kind, including children with HIVE into the sample of a group of children with spastic diplegia it does open the door for further research in this area. An aspect to consider when looking at the two different causes of spastic diplegia is that the children with HIVE fall into a very new subset of children for whom the appropriate treatment practices are relatively unknown. This could have resulted in limited management initially, with a greater potential to change once intervention occurred. Overall there were some differences with regard to the amount of functional change and the change in gait pattern at T2, but the outcomes at T3 were very similar. Further research will need to be done directly comparing the two groups, in a larger study, to truly understand the differences in the outcomes.

The main limitation of the study was the small sample size. One of the factors contributing to this is that SEMLS is a relatively new management approach for children with spastic diplegia accessing health care at a large academic hospital in South Africa. As the service continues to be offered at the hospital, the service will grow and the study can be broadened. The study was the first of its kind in this setting, therefore the aim was not to change the current service but to determine whether the current service was appropriate. The physiotherapy received was not standardised and it is difficult to determine what effect this had on the outcomes. Further limitations were the omission of a quality of life outcomes measure as well as the short follow-up period, ideally at least two years.

\section{Conclusion}

The results of this study indicate that the early outcomes of SEMLS in a South African setting, with scarce resources, are similar to those seen in developed countries. It may also be possible to use the same SEMLS treatment principles seen in the management of children with CP for children with static HIVE. Further follow-up is, however, needed in both of these areas.

\section{Compliance with ethical standards}

Informed consent from the Ethics Committee of the University of the Witwatersrand was obtained prior to commencement of this project (clearance number M120907).

\section{References}

1. Thomason P, Selber P, Graham K. Single event multilevel surgery in children with bilateral spastic cerebral palsy: a 5 year prospective cohort study. Gait and Posture 2013;37:23-38.

2. Harvey A, Rosenbaum P, Hanna $S$, et al. Longitudinal changes in mobility following single-event multilevel surgery in ambulatory children with cerebral palsy. Journal of Rehabilitation Medicine 2012;44:137-43.

3. Rutz E, Tirosh O, Thomason P, et al. Stability of the Gross Motor Function Classification System after single-event multilevel surgery in children with Cerebral Palsy. Developmental Medicine and Child Neurology 2012;54:1109-13.

4. Godwin E, Spero CR, Nof L, et al. Cerebral palsy and single-event multilevel sugery; Is there a relationship between level of function and intervention over time? Journal of Paediatric Orthopaedics 2009;29:910-15

5. Narayanan U. Management of ambulatory cerebral palsy: an evidence-based review. Journal of pediatric orthopaedics 2012;32:172-81.

6. Bischof F. Single event multilevel surgery in cerebral palsy: a review of the literature. South African Orthopaedic Journal 2010:30-33.

7. Hannah SE, Bartlett DJ, Rivard LM, et al. Reference curves for the gross motor function measure: percentiles for clinic description and tracking over time for children with cerebral palsy. Physical Therapy 2008;88:98-607.

8. Beckung E, Carlsson G, Carlsdotter S, et al. The natural history of gross motor development in children with cerebral palsy aged 1 to 15 years. Developmental Medicine and Child Neurology 2007;49:751-56. 
9. Thomason P, Baker R, Dodd K, et al. Single-event multilevel surgery in children with spastic diplegia; a pilot randomised controlled trial. The Journal of Bone and Joint Surgery 2011;93:451-60.

10. Rodda J, Graham K, Nattrass G, et al. Correction of severe crouch gait in patients with spastic diplegia with the use of multilevel orthopaedic surgery. Journal of Bone and Joint Surgery 2006;88:2653-64.

11. Harvey A, Graham K, Morris ME, et al. The functional mobility scale: ability to detect change following single event multilevel surgery. Developmental Medicine and Child Neurology 2007;49:603-607.

12. Khan MA. Outcome of single-event multilevel surgery in untreated cerebral palsy in a developing country. Journal of Bone and Joint Surgery [Br] 2007;89:1088-91.

13. Donald KA, Walker KG, Kilborn T, et al. HIV Encephalopathy: pediatric case series description and insights from the clinical coalface. AIDS Research and Therapy 2015;12:1-10.

14. Lowenthal ED, Bakeera-Kitaka S, Marukutira T, et al. Perinatally acquired HIV infection in adolescents from sub-Saharan Africa: A review of emerging challenges. Lancet Infectious Diseases 2014;14:627-39.

15. Baillieu N, Potterton J. The extent of delay of language, motor, and cognitive development in HIV-positive infants. JNPT 2008;32:118-21.

16. Govender R, Eley B, Walker K, et al. Neurologic and neurobehavioral sequelae in children with Human Immunodeficiency Virus (HIV-1) infection. Journal of child neurology 2001;26:1355-64.

17. Hilburn N, Potterton J, Stewart. Paediatric HIV encephalopathy in sub-Saharan Africa. Physical Therapy Reviews 2010;15:410-17.

18. Viehweger E, Zurcher Pfund L, Helix M, et al. Influence of clinical and gait analysis experience on reliability of observational gait analysis. Annals of Physical and Rehabilitation Medicine 2010;53:535-46.

19. Ong AM, Hillman SJ, Robb JE. Reliability and validity of the Edinburg Visual Gait Score for cerebral palsy when used by inexperienced observers. Gait and Posture 2008;28:323-26.

20. Debuse D, Brace H. Outcome measures of activity for children with cerebral palsy: a systematic review. Pediatric Physical Therapy 2011;23:222-31.

21. Russel DJ, Rosenbaum PL, Avery LM, Lane M. Gross Motor Function Measure (GMFM-88 \& GMFM-66) User's mannual 2002. Plymouth: Mac Keith Press.

22. Oeffinger D, Bagley A, Rogers S, et al. Outcome tools used for ambulatory children with cerebral palsy: responsiveness and minumum clinically important differences. Developmental Medicine and Child Neurology 2008;50:918-25.

23. Gupta S, Raja K. Responsiveness of Edinburgh Visual Gait Score to orthopaedic surgical intervention of the lower limbs in children with cerebral palsy. American Journal of Physical Medicine and Rehabilitaton 2012;91:761-67.

24. Read HS, Hazlewood ME, Hillman SJ et al. Edinburgh visual gait score for use in cerebral palsy. J Pediatr Orthop 2003;23:296-301.

25. Gorton III GE, Abel MF, Oeffinger DJ, et al. A prospective cohort study of the effects of lower extremity surgery on outcome measures in ambulatory children with cerebral palsy. Journal of Paediatric Orthopaedics 2009;29:903-909. 